\title{
Performance Analysis of a Multimedia CDMA System Using Dynamic Rate Control
}

\author{
Seung Sik Choi \\ Computer Eng. University of Incheon, \\ 177 Dohwa-dong Nam-gu Incheon, Korea 402-749, \\ PH: +82-32-770-8498, FAX: +82-32-766-6894 \\ sschoi@incheon.ac.kr
}

\begin{abstract}
In this paper, we propose an effective access control scheme in an voice/video/data integrated CDMA system. The proposed scheme schedules the transmission of data traffic during low voice/video traffic density period. In addition, to guarantee the delay requirements of data traffic, the transmission rate control of video traffic is triggered when the access delay of data traffic exceeds delay requirements. Numerical results show that outage probability is decreased by allowing the delay of data traffic and decreasing the transmission rates of video traffic.
\end{abstract}

\section{Introduction}

Recently, researches on an integrated voice/data CDMA system are performed. In reference [1], the power resource management concept was introduced and the feasible condition was derived. In reference [2], the capacity for data calls can be increased by scheduling data transmissions during low voice load and curtailing data transmissions when the voice traffic is heavy. This control scheme was extended and the prediction control scheme was suggested[3]. To support multimedia traffic in next generation systems, it is required to control resources in reverse links[4]. In reference [5], the transmission rate control of video traffic over wireless channels is proposed. Therefore, the increase of delay sensitive data traffic such as internet web service, requires to control not only data traffic but also video traffic. In this paper, we propose an effective access control scheme using delay and transmission control.

\section{Proposed Access Control Scheme}

The main characteristics of the proposed scheme is to control the delay of data traffic and the transmission rates of video traffic. Delay-based access control(DAC) scheme enables data traffic to be transmitted during inactive period of voice/video traffic. But, this scheme can't guarantee the quality of service(QoS) of data traffic because data traffic has larger delay in the case of heavy traffic condition. Due to this reason, it is required that video traffic should also be controlled when the delay requirements of data traffic can't be guaranteed. For video 
traffic, rate control scheme changes the transmission rate of video codec. For example, if the access delay of data traffic exceeds the access delay requirement, it triggers the transmission rate control of video traffic. Then, the station decreases the transmission rates of video traffics according to feedback information. In this paper, the access control scheme is called Delay and Transmission-based access control(DTAC) scheme.

\section{Performance Analysis}

Let the transmission rate of voice/video/data be $r_{u}, r_{v}$ and $r_{w}$ and the $E_{b} / N_{o}$ requirement of each class be $\gamma_{u}, \gamma_{v}$ and $\gamma_{w}$. The number of voice/video/data users are $K_{u}, K_{v}$ and $K_{w}$, respectively. If mobile stations in each class have the same transmission rate and BER requirement, the feasibility condition in n-th slot can be obtained as

$$
S(n)=\frac{U(n)}{a_{u}}+\frac{V(n)}{a_{v}}+\frac{W(n)}{a_{w}}<1
$$

where

$$
a_{u}=1+\frac{B}{r_{u} \gamma_{u}}, a_{v}=1+\frac{B}{r_{v} \gamma_{v}}, a_{w}=1+\frac{B}{r_{w} \gamma_{w}}
$$

Here $B$ is the total bandwidth. Based on the number of packets in the current slot, the permission probability for data traffic in the next slot is computed so that the target outage probability could be met.

Define $R(u, v)$ as the number of data users which are allowed in the next slot based on current active voice and video users. We can obtain $R(u, v)$ based on equation (1), the access probability of data traffic in next $(n+1)$ th slot can be calculated as following.

$$
p(n+1)=\max \left\{p \in[0,1] \mid \sum_{j=0}^{\lfloor R(u, v)\rfloor}\left(\begin{array}{c}
K_{w} \\
j
\end{array}\right) p^{j}(1-p)^{K_{w}-j}=\delta\right\}
$$

where $1-\delta$ is the outage probability requirements(normally 0.01 ). If the delay requirement of data traffic is $D_{\max }$ slot, the access probability of data users[4], $p_{\min }$, can be computed by

$$
p_{\min }=\frac{1}{1+D_{\max }}
$$

If $p(n+1)$ is greater than $p_{\text {min }}$, delay-based access control can be used. The minimum number of data users, $N_{w, \min }$, is given by

$$
N_{w, \text { min }}=\min \left\{k \in\left(0, K_{w}\right) \mid \sum_{j=0}^{k}\left(\begin{array}{c}
K_{w} \\
j
\end{array}\right) p_{\min }^{j}\left(1-p_{\min }\right)^{K_{w}-j}=\delta\right\}
$$


Let us define $a_{v}^{\prime}$ as the modified value of $a_{v}$, when the transmission rate control is applied. From (1), $a_{v}^{\prime}$ can be given by

$$
a_{v}^{\prime}>\frac{V(n)}{1-\frac{U(n)}{a_{u}}-\frac{N_{w, \min }}{a_{w}}}
$$

The transmission ratio of video traffic, $q(n+1)$, where $0 \leq q \leq 1(1$ : normal transmission, 0 : no transmission), is computed from (2) as following

$$
q(n+1)=\left\{\begin{aligned}
\frac{B}{r_{v} \gamma_{v}\left(a_{v}^{\prime}-1\right)}, & \text { if } p(n+1)<p_{\text {min }} \\
1, & \text { otherwise }
\end{aligned}\right.
$$

If the access probability of data traffic, $p(n+1)$, is lower than minimum value, $p_{\min }$, video transmission control is triggered.

\section{$4 \quad$ Numerical Results}

Consider a CDMA system with three classes of service such as voice, video and data. Each traffic has ON/OFF characteristics. The Eb/No, which is defined for service characteristics of voice, video and data traffic, is 6,7 and 8 $\mathrm{dB}$ respectively. The system bandwidth is $32 \mathrm{Mhz}$ and minimum access delay in DTAC scheme is constrained by $p_{\min }=0.5$. Based on these parameters, performances of DTAC scheme proposed in this paper is compared with those of DAC(delay-based access control) scheme and no access control scheme. The

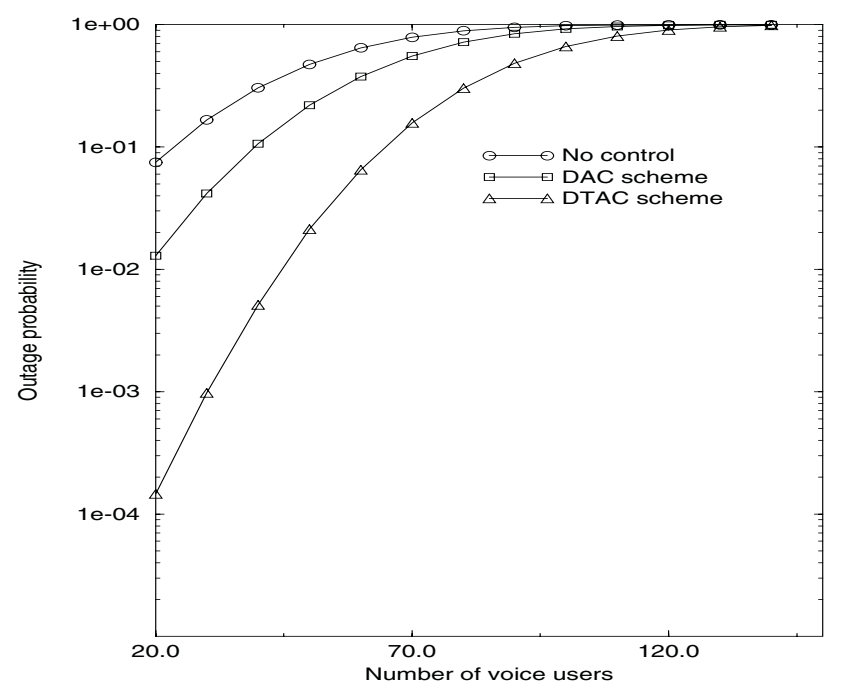

Fig. 1. Outage probability vs. number of voice users(when $K_{v}=7, K_{w}=10$ and $E b / N_{o}$ of data $=8 \mathrm{~dB})$ 


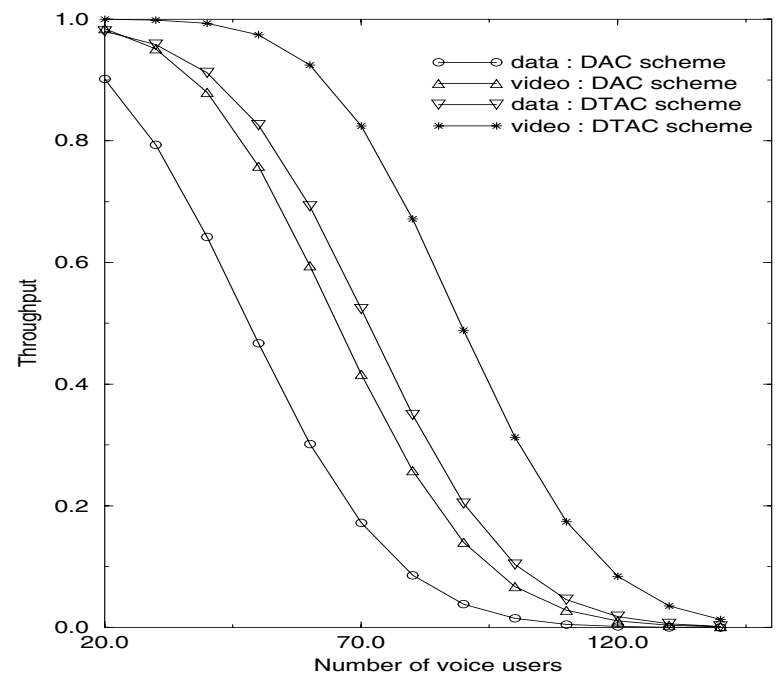

Fig. 2. Throughput vs.number of voice users(when $K_{v}=7, K_{w}=10$ and $E b / N o$ of data $=8 \mathrm{~dB})$

outage probability of three control schemes is shown in Fig. 1. It is shown that DTAC scheme has lower outage probability compared with two other schemes because it controls access of data and video traffics according to the traffic condition. The data/video throughput is shown in Fig.2. The data throughput in DTAC scheme is increased because the outage probability is decreased by the transmission rate control of video traffic. It is noted that the video throughput of DTAC scheme is increased even though the transmission rates of video traffics is decreased in heavy traffic condition. This result is due to the fact that the outage probability is decreased by DTAC scheme. It is shown that we could get more advantages by decreasing transmission rates of video traffics.

\section{References}

1. A.Sampath, P.S.Kumar, and J.M. Holtzman, "Power control and resource management for a multimedia wireless CDMA system," in proc. PIMRC'95, Toronto, Canada, Sept. 1995.

2. N.B. Mandayam and J.M. Holtzman," Analysis of a simple protocol for short message data service in an integrated voice/data CDMA system," in Proc. IEEE MILCOM'95, San Diego, CA, Nov. 1995.

3. Ashwin Sampath, Jack M. Holtzman, "Access Control of Data in Integrated Voice/Data CDMA Systems : Benefits and Tradeoffs," IEEE Journal of Selected Areas in Communications, Vol.15, No.8, pp.1511-1526, October 1997.

4. V.Huang and W.Zhuang, "QoS-Oriented Access Control for 4G Mobile Multimedia CDMA Communications," IEEE Communication Magazine, pp.118-125, March 2002.

5. Chi-Yuan Hsu, Antonio Ortega ,and M. Khansari "Rate Control for robust video transmission over burst-error wireless channels," IEEE Journal of Selected Areas in Communications, Vol.17, No.5, pp.756-773, May 1999. 